\title{
Impaired In Vivo Synaptic Plasticity in Dentate Gyrus and Spatial Memory in Juvenile Rats Induced by Prenatal Morphine Exposure
}

\author{
Lei Niu, ${ }^{1,2}$ Bing Cao, ${ }^{1,2}$ Hong Zhu, ${ }^{1,2}$ Bin Mei, ${ }^{3}$ Ming Wang, ${ }^{2}$ \\ Yupeng Yang, ${ }^{2}$ and Yifeng Zhou ${ }^{1,2,4 *}$
}

\begin{abstract}
Prenatal morphine exposure induces neurobiological changes, including deficits in learning and memory, in juvenile rat offspring. However the effects of this exposure on hippocampal plasticity, which is critical for learning and memory processes, are not well understood. The present study investigates the alterations of spatial memory and in vivo hippocampal synaptic plasticity in juvenile rats prenatally exposed to morphine. On gestation days 11-18, pregnant rats were randomly chosen to be injected twice daily with morphine or saline. Each juvenile offspring (postnatal day 22-31) performed one two-trial Y-maze task to evaluate spatial memory. Afterwards, the in vivo field excitatory postsynaptic potential (fEPSP) and population spike (PS) were recorded in the perforant path dentate gyrus (DG) pathway in the hippocampus. Prenatal morphine exposure reduced depotentiation (DP), but not longterm potentiation (LTP), of the EPSP slope. However, both LTP and DP of the EPSP slope were depressed in prenatal morphine-exposed juvenile offspring. The morphine group also showed poorer performance for the Y-maze task than the control group. Depressed PS LTP, but not EPSP LTP, in the morphine group suggested that prenatal morphine exposure changed GABAergic inhibition, which mediates EPSP-spike potentiation. Then a loss of GABA-containing neurons in the DG area of the morphine group was observed using immunohistochemistry. Taken together, our results suggest that prenatal morphine exposure impairs the juvenile offspring's dentate synaptic plasticity and spatial memory, and that decreased GABAergic inhibition may play a role in these effects. These findings might contribute to an explanation for the cognitive deficits in children whose mothers abuse opiates during pregnancy. $\odot 2008$ WileyLiss, Inc.
\end{abstract}

\footnotetext{
${ }^{1}$ Hefei National Laboratory for Physical Sciences at Microscale and School of Life Science, University of Science and Technology of China, Hefei, Anhui, People's Republic of China; ${ }^{2}$ Department of Neurobiology and Biophysics, School of Life Science, University of Science and Technology of China, Hefei, Anhui, People's Republic of China; ${ }^{3}$ Department of Biology, School of Basic Medical Science, Anhui Medical University, Hefei, Anhui, People's Republic of China; ${ }^{4}$ State Key Laboratory of Brain and Cognitive Science, Institute of Biophysics, Chinese Academy of Science, Beijing, People's Republic of China

Grant sponsor: National Natural Science Foundation of China; Grant number: 30470561; Grant sponsor: National Basic Research Program; Grant number: 2006CB500804.

Abbreviations used: DG, dentate gyrus; DP, depotentiation; EPSCs, excitatory postsynaptic currents; fEPSP, field excitatory postsynaptic potential; GABA, gamma-aminobutyric acid; HFS, high-frequency stimulation; I/O, input/output function; IPI, interpulse interval; ITI, intertrial interval; LFS, low-frequency stimulation; LTD, long-term depression; LTP, long-term potentiation; PS, population spike(s).

*Correspondence to: Yifeng Zhou, School of Life Science, University of Science and Technology of China, Hefei, Anhui 230027, People's Republic of China. E-mail: zhouy@ustc.edu.cn

Accepted for publication 14 November 2008

DOI 10.1002/hipo.20540

Published online in Wiley InterScience (www.interscience.wiley.com).
}

KEY WORDS: depotentiation; development; hippocampus; LTP; prenatal drug exposure

\section{INTRODUCTION}

The number of opiate users has risen to around 16 million people or $0.4 \%$ of the world's population in the age range of 15-64 (UNODC, 2006). Cognitive problems of the children whose mothers abuse opiates during pregnancy are now of major concern. Opiates, such as morphine, can cross the placenta to affect the development of the central nervous system and thereby result in neurobiological changes in juvenile offspring. These changes include alterations in play and social behavior (Hol et al., 1996; Niesink et al., 1996, 1999), sensitivity in morphine-induced analgesia (O'Callaghan and Holtzman, 1976; Kirby et al., 1982; Chiou et al., 2003), seizure susceptibility (Vathy et al., 1998; Veliskova et al., 1999), spinal cord volume (Kirby, 1983), opioid mu receptors (Bhat et al., 2006a), and brain cyclin-dependent kinase 5 activity (Bhat et al., 2006b).

Prenatal morphine exposure also impairs learning and memory in juvenile rats (Yang et al., 2003, 2006). Synaptic plasticity in the hippocampus is critical for learning and memory processes (Morris et al., 1986; Morris, 1989; Silva et al., 1992). However, the effects of this exposure on hippocampal plasticity in juvenile rats are not well understood. Yang et al. (2003) reported that prenatal morphine exposure attenuated long-term depression (LTD) and depotentiation (DP), but not LTP, of excitatory postsynaptic currents (EPSCs) in the CA1 area of hippocampal slice of juvenile rats. However, in the hippocampal CA1 area of adult rats, prenatal morphine exposure depressed long-term potentiation (LTP) of the excitatory postsynaptic potential (EPSP) in vitro (Velisek et al., 2000). The discrepancy may be due to the difference in the age of offspring. In the adult hippocampal dentate gyrus (DG) area, prenatal morphine exposure reduced LTP in anesthetized rats (Sarkaki et al., 2008), or late-LTP $(>24 \mathrm{~h})$ in freely moving rats 
(Villarreal et al., 2008). However, few studies have been carried out in the DG area of juvenile rats.

The characteristic response of DG granule cells to perforant path stimulation consists of a positive-going EPSP with a superimposed negative-going field PS (Lomo, 1971a,b). In the present study, we investigated LTP and DP of both EPSP slope and PS amplitude in vivo in the DG area of prenatally salineand morphine-exposed juvenile rat offspring. We also evaluated spatial memory in these offspring using a two-trial Y-maze test (Dellu et al., 1997), since the DG area of the hippocampus has been associated with spatial memory (Colicos and Dash, 1996; Nilsson et al., 1999; Rubin et al., 1999). Finally we determined whether GABA-containing neurons in the DG area, which largely control the EPSP/spike relationship (Tomasulo et al., 1991; Marder and Buonomano, 2004) and the functions of dentate granule cells (Coulter and Carlson, 2007), would be alternated by prenatal morphine exposure. These studies suggest that prenatal morphine exposure decreased in vivo synaptic plasticity and the number of GABA-containing neurons in the DG area of juvenile rat offspring with deficient spatial memory.

\section{MATERIALS AND METHODS}

\section{Prenatal Treatment}

Adult female and male Sprague-Dawley rats were obtained from Anhui Experimental Animal Center and maintained on a $12 \mathrm{~h}$ light/dark cycle (9:00-21:00 h) with free access to food and water. All animal treatments were strictly in accordance with the National Institutes of Health Guide for the Care and Use of Laboratory Animals. The estrous cycle phases of female rats were determined according to the cell types observed in the vaginal smear for 10 days (Marcondes et al., 2002). The female rats with normal estrous cycles were mated at night during their proestrus or estrus phase. The presence of vaginal plug or sperm cells in vaginal smear the next morning and a high rate of body weight gain in the next 10 days were used as the indices for pregnancy. Pregnant rats were randomly injected with morphine (morphine sulfate, $10 \mathrm{mg} / \mathrm{ml}$, obtained from the Anhui Food \& Drug Administration, China) or saline twice daily at 09:00 and 21:00 $\mathrm{h}$ on gestation days 11-18. The dose of the first three morphine injections was $5 \mathrm{mg} / \mathrm{kg}$ and the dose for all subsequent injections was $10 \mathrm{mg} / \mathrm{kg}$ (Vathy et al., 1985). All injections were administered subcutaneously (s.c.).

\section{Postnatal Treatment}

The day of birth was designated postnatal day (PND) 0 . On PND1, pups were sexed and weighed. The injection paradigm did not alter the number of pups per litter and the body weight of the offspring as described in previous studies (Vathy et al., 1985; Velisek et al., 2000; Riley and Vathy, 2006). On
PND 21, pups were weaned and housed in groups with free access to food and water. Experiments were performed in prenatally saline- (control group) and morphine-exposed (morphine group) juvenile offspring (PND 22-31).

\section{Stimulation and Recording}

In each recording session, rats were anesthetized with urethane $(20 \%, 1.2 \mathrm{~g} / \mathrm{kg}$, i.p.) and were then mounted in a stereotaxic apparatus. The skull was exposed and the rectal temperature was monitored and maintained at $37^{\circ} \mathrm{C} \pm 0.5^{\circ} \mathrm{C}$ by a homeothermic blanket. A concentric bipolar electrode (FHC, Bowdoinham, ME) was placed in the perforant path (coordinates with the skull surface flat: $7.0-8.0 \mathrm{~mm}$ posterior to bregma, $4.2 \mathrm{~mm}$ lateral to the midline, $2.8 \mathrm{~mm}$ ventral). A glass micropipette recording electrode (tip size $3 \mu \mathrm{m}$, with an impedance of 2-3 $M \Omega$ while filled with saline) was lowered into the DG (coordinates: $3.5 \mathrm{~mm}$ posterior to bregma, 2.0 $\mathrm{mm}$ lateral to the midline) until the maximal response to perforant path stimulus was observed (3.0-3.4 mm ventral).

Extracellular evoked responses were obtained from the dentate granule cell population in response to electrical stimulation of the perforant path. The electrical stimulus was driven by computer-controlled ITC-18 data acquisition interface (Instrutech Corp., NY), and an isolator unit (WPI, Sarasota, FL) was used to minimize the artifact. The duration of biphasic pulse was $100 \mu \mathrm{s}$. The intensity of the test stimuli was adjusted to evoke about $50 \%$ of the maximum response of population spike (PS) amplitude.

\section{Input/Output Function}

I/O curves were generated by systematic variation of the stimulus current $(0.1-1.0 \mathrm{~mA})$ to evaluate synaptic potency. Stimulus pulses were delivered at $0.1 \mathrm{~Hz}$ and three responses at each current level were averaged.

\section{Paired-Pulse Response}

Pairs of identical stimuli were delivered to the perforant path with interpulse intervals (IPIs) ranging from 25 to $300 \mathrm{~ms}$. The size of the second EPSP slope or PS amplitude was then expressed as a percentage of the first. A value less than $100 \%$ indicated paired-pulse depression, whereas a value greater than $100 \%$ indicated paired-pulse facilitation. An averaged value was calculated from three successive responses with $20 \mathrm{~s}$ between each stimulus pair.

\section{Long-Term Potentiation and Depotentiation}

In this study, LTP and DP were performed on each rat. LTP was induced after stable baseline recording for at least $30 \mathrm{~min}$ by delivery of high-frequency stimulation (HFS; 11 trains of 10 pulses at $250 \mathrm{~Hz}$ separated by $1 \mathrm{~s}$ ). Test pulses were applied for $60 \mathrm{~min}$ to measure LTP. After another $80 \mathrm{~min}$ for break, following 10 min baseline recording, DP was induced by lowfrequency stimulation (LFS; 900 pulses at $1 \mathrm{~Hz}$ for $15 \mathrm{~min}$ ). The test pulses were delivered at $0.05 \mathrm{~Hz}$ throughout the 
experiment. The protocol is illustrated in Figure 3. At the end of each recording session, small electrolytic lesions (1 mA, $10 \mathrm{~s})$ were made to permit histological verification of the tip position of the electrodes.

\section{Y-Maze Test}

In the present study, the rats were evaluated for spatial memory in one wooden Y-maze before electrophysiological experiment. The maze was lacquered black, and consisted of three arms with an angle of $120^{\circ}$ between each two arms. Each arm was $16 \mathrm{~cm} \times 50 \mathrm{~cm} \times 30 \mathrm{~cm}$ (width $\times$ length $\times$ height). The three identical arms were randomly designated: Start arm, in which the rats started to explore (always open), Novel arm, which was blocked at the first trial, but open at the second trial, and Other arm (always open).

The maze was placed in a sound-attenuated room under dim illumination $(\approx 5$ lux on the floor of maze). The floor of the maze was covered with sawdust, which was mixed after each individual trial to eliminate olfactory stimuli. Visual cues were placed on the walls of the maze. The movements of the rats were recorded by a camera over the maze. The video was stored in a computer and later analyzed by a person who was blind to the animal groups.

The Y-maze test consisted of two trials separated by an intertrial interval (ITI) to assess spatial recognition memory. The first trial (training) lasted for $10 \mathrm{~min}$ and allowed the rat to explore only two arms (Start arm and Other arm) of the maze, with the third arm (Novel arm) being blocked. After a $2 \mathrm{~h} \mathrm{ITI,}$ the second trial (retention) was conducted. The rat was placed back in the maze in the same starting arm, with free access to all three arms for $2 \mathrm{~min}$. The first arm visited, the number and duration of explorations of each arm were recorded during the second trial (Dellu et al., 1997).

\section{Immunohistochemistry}

Rat offspring (PND 26) were deeply anesthetized with urethane and perfused transcardially with chilled physiological saline till the liver became pale, immediately followed by $0.1 \mathrm{M}$ phosphate buffer (PB) containing $4 \%(\mathrm{w} / \mathrm{v})$ paraformaldehyde and $1.25 \%(\mathrm{w} / \mathrm{v})$ glutaraldehyde. Brains were removed and stored in the same paraformaldehyde solution overnight, and then the samples were washed with water, dehydrated in ethanol, transparentized with xylene, and embedded in paraffin. Coronal sections of $6 \mu \mathrm{m}$ thickness were cut and mounted for immunohistochemical staining on gelatin-coated microscope slides. The hippocampal sections were washed with $0.01 \mathrm{M}$ PBS phosphate-buffered saline (PBS), deparaffinized in xylene, hydrated through a graded series of ethanol, and washed for $10 \mathrm{~min}$ in distilled water. After washing with PBS, the sections were incubated in PBS containing $0.3 \% \mathrm{H}_{2} \mathrm{O}_{2}$ and $1 \%$ Triton $\mathrm{X}-100$ for $30 \mathrm{~min}$ at room temperature to quench endogenous peroxidase activity. Then the sections were washed with PBS, incubated in 5\% goat serum, and then in mouse anti-GABA serum $\left(37^{\circ} \mathrm{C}\right.$ for $60 \mathrm{~min}, 1: 200$ dilution; A0310, Sigma, St. Louis, MO). After incubation at $4{ }^{\circ} \mathrm{C}$ overnight, sections were washed with PBS, incubated in biotinylated goat antimouse IgG antibody (at $37^{\circ} \mathrm{C}$ for $60 \mathrm{~min}, 1: 1,000$ dilution; B0529, Sigma, St. Louis, MO), washed with PBS, incubated in the avidin-biotin complex (at $37^{\circ} \mathrm{C}$ for $60 \mathrm{~min}, 1: 200$ dilution; PK6100, Vector Laboratories, Burlingame, CA) in PBS After washes with $\mathrm{PBS}$, the sections were visualized by adding $0.03 \%$ $\mathrm{H}_{2} \mathrm{O}_{2}$ to a solution containing $0.05 \% 3,3^{\prime}$-diaminobenzidine tetrahydrochloride (DAB; D5637, Sigma, St. Louis, MO), dehydrated in ethanol, cleared in xylene, and mounted in Entellan.

\section{Data Analysis}

The EPSP slope was calculated from EPSP onset $0.5 \mathrm{~ms}$ to the beginning of the negative component of PS. The PS amplitude was measured by averaging the distance from the negative peak to leading and following positive peak (Burdette and Gilbert, 1995). The responses in every 5 min were averaged and were normalized to pretetanus baseline values. The amplitudes of LTP were calculated by using the percentage of the means for the $60 \mathrm{~min}$ post-HFS compared with pretetanus baseline data, the amplitudes of late-LTP were calculated by using the percentage of the 10-min pre-LFS data compared with pretetanus baseline data, and the amplitudes of DP were calculated by using the percentage of the means for 10 to $45 \mathrm{~min}$ post-LFS compared with the 10-min pre-LFS baseline data. In Y-maze test, the duration of arm visits in the second trial was as a spatial recognition memory measure. For immunohistochemistry analysis, the number of dentate GABA-containing neurons for each animal was evaluated in at least five sections obtained every 10th section. Since GABA-containing neurons mostly exist within or directly beneath granule cell layer (Robert and Sloviter, 1987), the neurons in granule cell layer and subgranular zone were counted.

The differences between groups were tested by repeated measures ANOVA. The sex-special effects were tested by twoway ANOVA (Group $\times$ Gender). When the duration of visits in the different arms were compared, arm (start, other, novel) was considered a within-group factor followed by post hoc (LSD) test. After analysis of ANOVA, some data were analyzed by Student's $t$-test for paired comparisons. Percentages of first choices in the novel arm were compared to chance $(50 \%)$ using the $\chi^{2}$ test. Statistical significance was set at the probability level of $P<0.05$.

\section{RESULTS}

Electrophysiological and behavioral experiments were performed in prenatally saline- (seven males and seven females) and morphine-exposed juvenile rats (seven males and six females) at age of PND 22-31. The average age was PND $27.1 \pm 0.9$ in the control group and PND $26.3 \pm 0.9$ in the morphine group $(t(25)=0.630, P=0.534)$. Immunohistochemical experiments were performed in prenatally saline- 
(three males and three females) and morphine-exposed juvenile rats (three males and three females) at age PND 26. In these experiments, neither main effect of gender nor the gender $X$ group interaction was significant (all n.s.).

\section{I/O Functions and Paired-Pulse Response}

Prenatal morphine exposure did not affect the $\mathrm{I} / \mathrm{O}$ curves in the DG area (all n.s.) measured by the EPSP slope (Fig. 1A), the PS amplitude (Fig. 1B), and the EPSP-PS plot (Fig. 1C). Paired-pulse response was shown in Figure 2. No significant difference was observed between the control $(n=14)$ and morphine groups $(n=13)$ in the paired-pulse response of the EPSP slope (Fig. 2B, all n.s.). Meanwhile, the paired-pulse response of the PS amplitude (Fig. 2C) has slight differences at long IPIs $(200 \mathrm{~ms}, t(25)=2.155, P<0.05 ; 300 \mathrm{~ms}, t(25)=$ 2.292, $P<0.05$ ).

These results suggested that prenatal morphine exposure does not affect the basal synaptic transmission of dentate granule cells in juvenile rats.

\section{Long-Term Potentiation and Depotentiation}

The amplitudes of LTP and DP in the DG area of juvenile offspring were shown in Figure 3. Prenatal morphine exposure had no effects on LTP of the EPSP slope (Fig. 3B). The LTP amplitude of the EPSP slope was $123 \% \pm 4 \%$ in the control group $(n=14)$ and $127 \% \pm 6 \%$ in the morphine group $(n=13, F(1,25)=0.352, P=0.559)$ at $60 \mathrm{~min}$ after HFS. After a break of $80 \mathrm{~min}$, the late-LTP amplitudes of the EPSP slope in two groups were similar (the control group, $118 \% \pm$ $8 \%, n=14$; the morphine group, $114 \% \pm 6 \%, n=12$, $F(1,24)=0.110, P=0.743)$. However, prenatal morphine exposure affected LTP of PS amplitude (Fig. 3C). The LTP amplitude of PS (at 60 min after HFS) decreased from 199\% \pm $19 \%(n=14)$ in the control group to $144 \% \pm 13 \%$ in the morphine group $(n=13, F(1,25)=4.798, P<0.05)$. The late-LTP amplitude of PS (at $140 \mathrm{~min}$ after HFS) was still significantly different between the control $(198 \% \pm 23 \%, n=$ 13) and morphine groups $(137 \% \pm 14 \%, n=12, F(1,24)$ $=4.698, P<0.05)$.

DP amplitudes of both the EPSP slope (Fig. 3B) and PS (Fig. 3C) were significantly depressed in the morphine group (EPSP slope, $F(1,24)=4.312, P<0.05$; PS amplitude, $F$ $(1,24)=6.005, P<0.05)$ after LFS $(900$ pulses at $1 \mathrm{~Hz})$. The corresponding means were $86 \% \pm 4 \%$ (EPSP slope) and $80 \% \pm 4 \%$ (PS amplitude) in the control group $(n=14)$ and $96 \% \pm 2 \%$ (EPSP slope) and $91 \% \pm 2 \%$ (PS amplitude) in the morphine group $(n=12)$.

\section{Y-Maze Test}

The ability of spatial memory, which is associated with the hippocampal DG area function, was tested by the Y-maze task just before electrophysiological experiment in each rat. The percentage of first visits in the novel arm in the control group was above chance $(50 \%)$ since 12 of 14 rats made their first choice
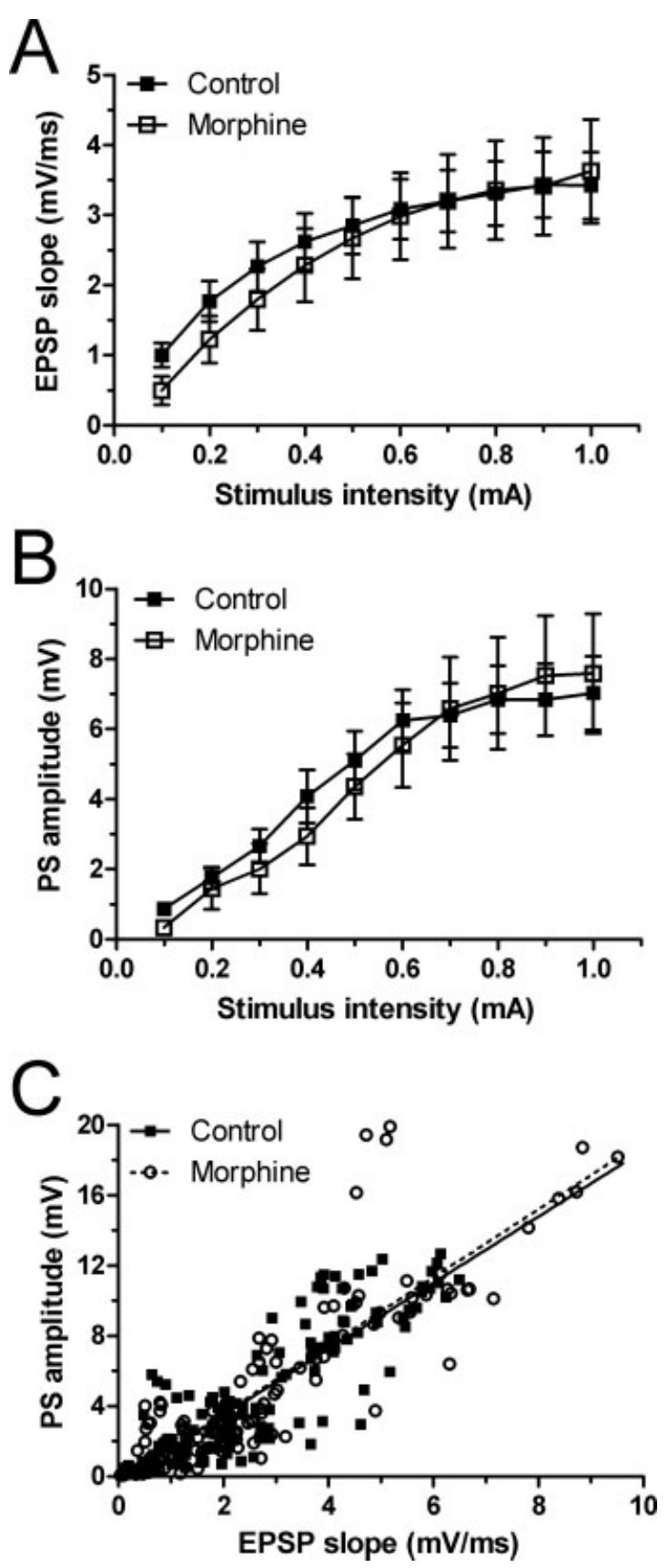

FIGURE 1. I/O curves (mean \pm SEM) of the EPSP slope (A) and PS amplitude (B) in the DG area in juvenile offspring (control group, $n=14$, and morphine group, $n=13$ ) as a function of stimulus intensity before induction of LTP. Panel C shows the PS amplitude as a function of the EPSP slope, and the solid and dim lines are regression lines fitting the data in the control $(n=14$, $\left.r^{2}=0.7313, P<0.001\right)$ and the morphine $\left(n=13, r^{2}=0.7814\right.$, $P<0.001)$ groups, respectively.

in the novel arm $\left(85.71 \% ; \chi^{2}=7.14, P<0.01\right)$. The morphine group, however, made their first choice in a random way as only 8 of 13 rats chose the novel arm $\left(61.54 \% ; \chi^{2}=\right.$ $0.692, P=0.405)$. As shown in Figure 4, the duration of visits in the novel arm was significantly increased compared with the start and other arms after $2 \mathrm{~h}$ ITI in the control group (effect 

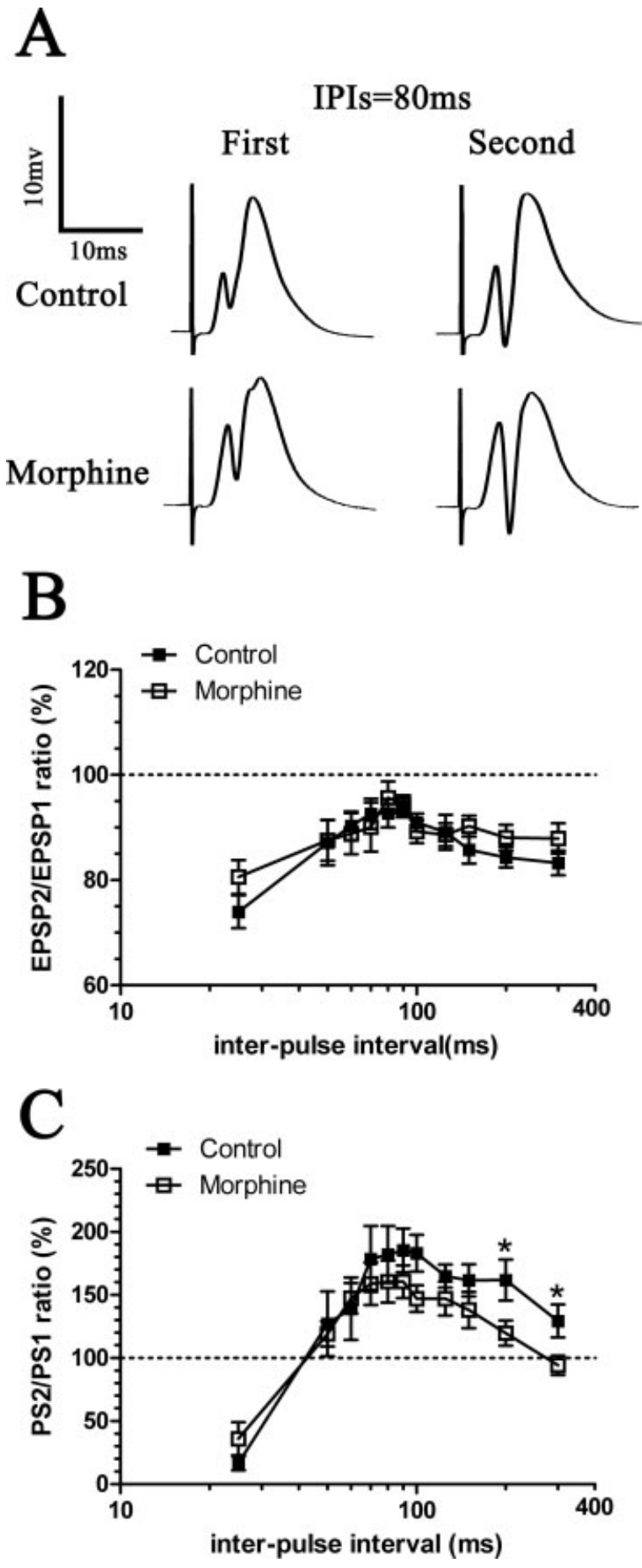

FIGURE 2. Paired-pulse response in the DG area of juvenile offspring at varying interpulse intervals of $25-300 \mathrm{~ms}$. A: Representative first and second waveforms in 80-ms IPIs. Each case was from a single experiment and averaged three times. B, C: Pairedpulse response (mean \pm SEM) of the EPSP slope (B) and the PS amplitude (C).The dashed line represents a ratio of one (EPSP2/ EPSP1 = 1 or PS2/PS1 = 1), where no inhibition or facilitation of the second response relative to the first response would occur. Asterisks indicate significant difference $(P<0.05)$ between prenatally saline- $(n=14)$ and morphine-exposed rats $(n=13)$.
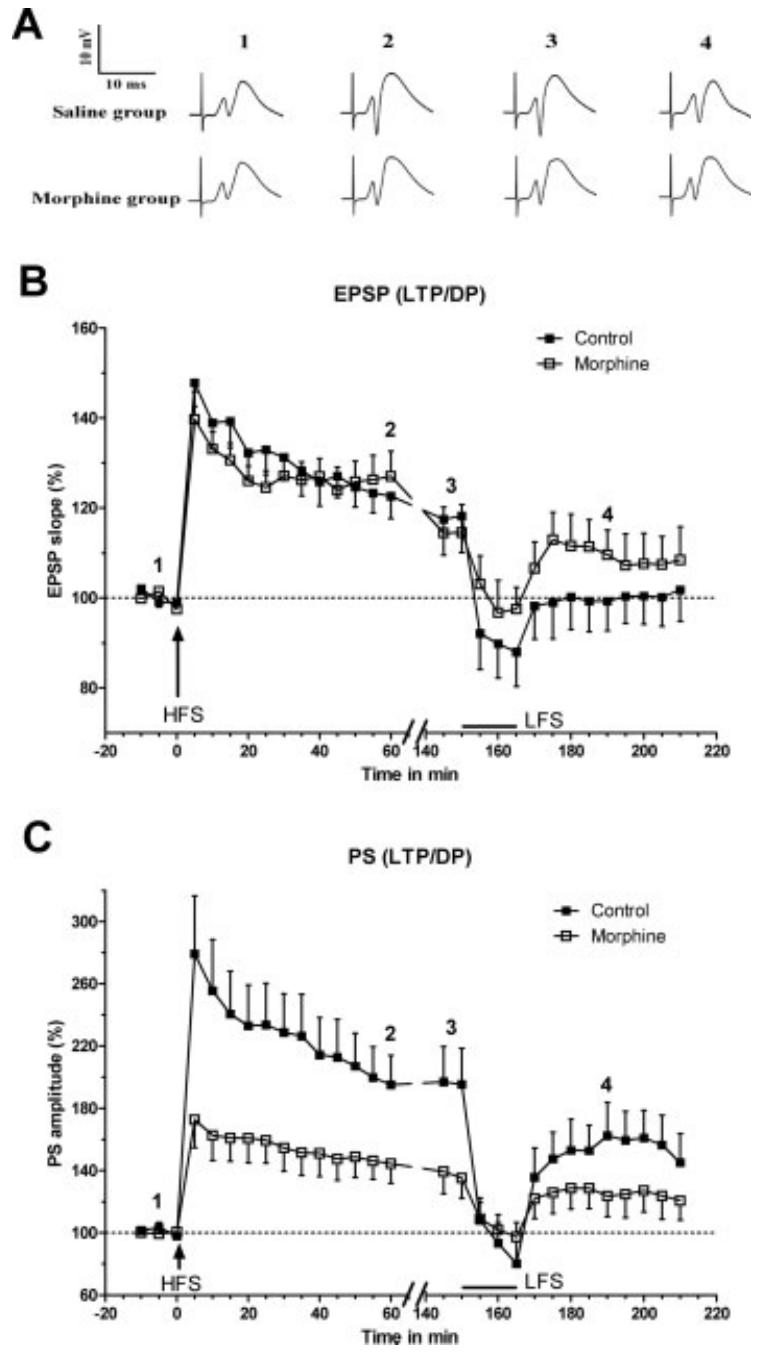

FIGURE 3. LTP and DP in the DG area of juvenile offspring. A: Representative waveforms before and after LTP/DP inductions in control and morphine groups. The waveforms in each case are from a single experiment. B, C: Effects of prenatal morphine exposure on LTP/DP (mean \pm SEM) of the EPSP slope (B) and PS amplitude (C) in juvenile offspring. At 60 min post-HFS, the LTP amplitude of the EPSP slope was $123 \% \pm 4 \%$ in the control $(n=14)$ and $127 \% \pm 6 \%$ in the morphine groups $(n=13)$ with no significant difference $(F(1,25)=0.352, P=0.559)$, while the LTP amplitude of the PS was $199 \% \pm 19 \%$ in the control group and was significantly depressed to $144 \% \pm 13 \%$ in the morphine group $(F(1,25)=4.798, P<0.05)$. After a break of $80 \mathrm{~min}$, there also was a significant difference in the late-LTP amplitude of PS (averaged $10 \mathrm{~min})$ between the control $(198 \% \pm 23 \%, n=14)$ and morphine groups $(137 \% \pm 14 \%, n=12, F(1,24)=4.698, P<$ $0.05)$, but not in the late-LTP amplitude of the EPSP slope (control group, $118 \% \pm 8 \%, n=14$; morphine group, $114 \% \pm 6 \%, n=$ $12, F(1,24)=0.110, P=0.743)$. After LFS, the DP amplitude was $86 \% \pm 4 \%$ (EPSP slope) and $80 \% \pm 4 \%$ (PS amplitude) in the control group $(n=14)$, and was significantly different with $96 \% \pm 2 \%$ (EPSP slope: $F(1,24)=4.312, P<0.05)$ and $91 \% \pm$ 2\% (PS amplitude: $F(1,24)=6.005, P<0.05)$ in the morphine group $(n=12)$, respectively. Each point represents the mean averaged in over $5 \mathrm{~min}$. Arrows indicate the application of HFS, and horizontal solid lines indicate the application of LFS. 


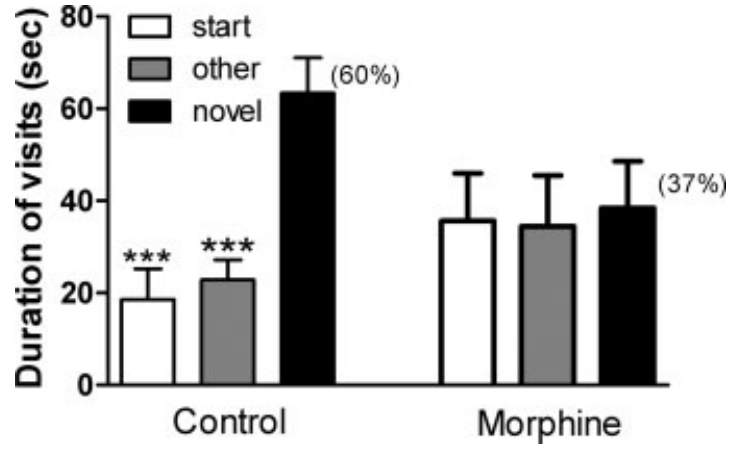

FIGURE 4. Effects of prenatal morphine exposure on total duration (mean \pm SEM) of Y-maze visits after $2 \mathrm{~h}$ ITI in juvenile rats (control group, $n=14$; and morphine group, $n=13$ ). The mean total duration for control rats to explore the novel arm was significantly higher than for the start or other arms. The percentage duration of visits in the novel arm with respect to the total duration of visits in the three arms are indicated in parentheses (chance level is $33 \%)$. Difference in performance of rat in the novel arm versus the start and other arms is indicated as ${ }^{* * *}(P<0.001)$.

of arm: $F(2,39)=15.114, P<0.001$; LSD: novel arm vs. start arm: $P<0.001$, novel arm vs. other arm: $P<0.001$, star arm vs. other arm: $P=0.636$ ). However, no arm difference of duration of visits was found in the morphine group (effect of arm: $F(2,36)=0.038, P=0.968)$. The average percentage of duration of visits in the novel arm was $60 \%$ in the saline group and decreased to $37 \%$ in the morphine group.

\section{GABA-Containing Neurons}

To determine whether the GABAergic inhibition, an important system to the EPSP/spike relationship and learning and memory, was altered by prenatal morphine exposure, GABAcontaining neurons in the DG area were counted by immunohistochemistry with DAB staining. As illustrated in Figure 5, prenatal morphine exposure significantly decreased the number of GABA-containing neurons in the DG area (the control group, $22.4 \pm 0.8, n=6$, Fig. $5 \mathrm{~A}$; the morphine group, $11.5 \pm 0.8$, $n=6$, Fig. 5B; $F(1,10)=94.931, P<0.001$, Fig. 5C).

\section{DISCUSSION}

In this study, we observed that prenatal morphine exposure depressed DP, but not LTP of the EPSP slope in vivo in hippocampal DG area of juvenile rats, which is consistent with previous work in vitro in CA1 area of 2 week old rats prenatally exposed to morphine (Yang et al., 2003). Interestingly, this exposure had different effects on PS amplitude. Both LTP and DP of the PS amplitude in the DG area were reduced. This exposure also impaired spatial memory and decreased the number of GABA-containing neurons in the DG area.

LTP in the hippocampus is composed of two independent components-a synaptic component and an EPSP-to-spike coupling component (Taube and Schwartzkroin, 1988). The

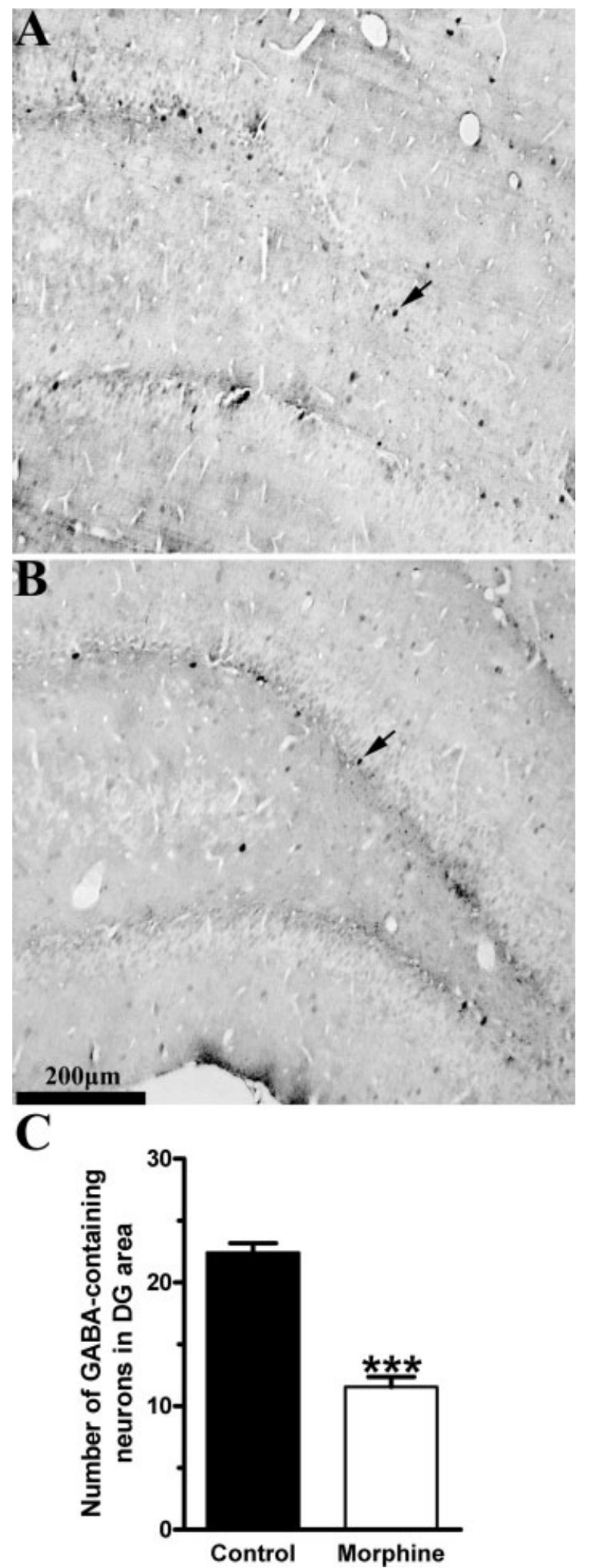

FIGURE 5. DAB staining for GABA-containing neurons in the hippocampal DG area of the control (A) and morphine groups (B) at PND 26; $\times 100$, scale bar $=200 \mu \mathrm{m}$. Arrows indicate example of labeled cells. Panel $\mathrm{C}$ shows the numbers of GABA-containing neurons (mean \pm SEM) in the control $(n=6)$ and morphine groups $(n=6)$. Significant difference $(P<0.001)$ from the control group is indicated as $* * *$. 
latter is also called EPSP-spike potentiation, which enhances the ability of an EPSP of a fixed slope to elicit spikes after HFS-induced LTP. In our study, PS LTP was depressed whereas EPSP LTP was not in the morphine group, suggesting that prenatal morphine exposure impaired EPSP-spike potentiation. EPSP-spike potentiation depends on the balance and timing of $\mathrm{GABA}_{\mathrm{A}}$ ergic inhibition (Marder and Buonomano, 2004). $\mathrm{GABA}_{\mathrm{A}} \mathrm{R}$ antagonists such as picrotoxin or bicuculline could block the EPSP-spike potentiation (Abraham et al., 1987; Lu et al., 2000). In addition, previous results showed that prenatal exposure to morphine decreased susceptibility to $\mathrm{GABA}_{\mathrm{A}}$-regulated seizures in juvenile and adult male rats (Schindler et al., 2000, 2001). Therefore, the depression of LTP in the PS but not in the EPSP may be due to changes of $\mathrm{GABA}_{\mathrm{A}}$ ergic inhibition. This hypothesis was partly supported by our immunohistochemical result, that a loss of GABA-containing neurons in the DG area was observed in juvenile offspring prenatally exposed to morphine.

No alterations of EPSP LTP measured over $1 \mathrm{~h}$ were observed in juvenile rats prenatally exposed to morphine. This result agrees with a previous study in adult freely moving rats (Villarreal et al., 2008). However, Sarkaki et al. reported that prenatal morphine exposure decreased EPSP LTP in the DG area of adult anesthetized rats (Sarkaki et al., 2008). The apparent discrepancy could be due to differences in morphine dose and exposure schedules, since we used the same injection paradigm as that of Villareal et al. (2008).

DP in the DG area was obtained induced by $1 \mathrm{~Hz}$ LFS after 150 min post-HFS in the present study. We also observed DP in the DG area with $1 \mathrm{~Hz}$ LFS being applied after $15 \mathrm{~min}$ post-HFS (data not shown here). These observations are in line with previous studies demonstrating DP could be found in the DG area of anesthetized rats following $1 \mathrm{~Hz}$ LFS after 55, 60, or 130 min post-HFS was applied (Kimura and Pavlides, 2000; She et al., 2005; Zhu et al., 2005; Wang et al., 2007). However, DP in the DG area could not be induced by 2 or $5 \mathrm{~Hz}$ LFS after $30 \mathrm{~min}$ post-HFS in anesthetized rats (Errington et al., 1995). On the other hand, DP in the DG area of freely moving rats could be induced by 3-7 Hz LFS within 5 min post-HFS (Abraham et al., 1996; Kulla et al., 1999; Kulla and Manahan-Vaughan, 2000, 2002, 2008; Manahan-Vaughan and Kulla, 2003; Straube and Frey, 2003; Klausnitzer et al., 2004), but not by $1 \mathrm{~Hz}$ post-HFS after $1,15,30$, or $60 \mathrm{~min}$ postHFS (Errington et al., 1995; Abraham et al., 1996). These results suggest that the time window of DP induction in the DG area may depend on the LFS frequency and level of wakefulness versus anesthesia.

Spatial memory deficits were found in prenatally morphineexposed juvenile rats in the Y-maze task. This result is consistent with previous work on memory deficits induced by prenatal morphine exposure in chicks (Che et al., 2005). Yang et al. (2003) have suggested that prenatal morphine exposure impaired the spatial learning and memory of juvenile rats (PND 28-31) in a Morris water maze task. However, the impairment occurred only in the first training day, and the morphine-treated and control groups displayed similar improvements over the 4 days of training. Thus this finding did not fully demonstrate the effects on spatial memory. The Y-maze task is based on the innate tendency of rats to explore novelty and does not require learning of a rule, so it is a specific and sensitive test of spatial recognition memory in rodents (Dellu et al., 1997, 2000). Thus our result, along with the work by Yang et al. (2003), indicates that spatial memory in juvenile rats is impaired by prenatal morphine exposure. This behavioral result could support our electrophysiological findings, since LTP of PS in the DG area has been reported to be associated with spatial memory (Mori et al., 2001; Huang et al., 2006).

Sex-specific changes reported in adult rats induced by prenatal morphine exposure include alterations in NE and opioid systems (Vathy and Katay, 1992; Vathy et al., 1994), endogenous opioid systems (Slamberova et al., 2004), and $\mu$-opioid receptor density in hippocampus (Slamberova et al., 2003) and motivation systems (Vathy et al., 2003), and learning and memory (Slamberova et al., 2001). Schindler et al. (2000) also reported that prenatal morphine exposure altered the susceptibility to bicuculline seizures in juvenile and adult rats in a sexand age-specific manner. However, using the same injection paradigm, we did not observe a sex-specific effect on impairment of synaptic plasticity in the hippocampal DG area in juvenile rats prenatally exposed to morphine. A similar result was reported in adult rat offspring, that LTP in the DG area of male and female offspring were not differently affected by prenatal morphine exposure (Sarkaki et al., 2008). Thus it appears that the impairments of synaptic plasticity in the DG area induced by prenatal morphine exposure were not sex-specific.

This report suggests that prenatal morphine exposure impairs hippocampal synaptic plasticity and spatial memory in juvenile offspring, and that decreased GABAergic inhibition may play a role in these effects. Although the connection between psychological and behavioral results in rodents and cognition in humans is not straightforward, our data may contribute to the understanding cognitive deficits in children whose mothers abuse opiates during pregnancy (Zuckerman and Bresnahan, 1991; van Baar et al., 1994). Further longitudinal research is necessary to discover how the GABAergic inhibition system contributes to the psychological and behavioral changes induced by prenatal morphine exposure.

\section{Acknowledgments}

The authors thank Dr. Ilona Vathy for her help on the rat model, Dr. Yuanye Ma and Dr. Jianhong Wang for their help on the Y-maze test, and Dr. Bing Hu for his help with immunohistochemical methods. They also thank Dr. Curtis L. Baker for polishing the English.

\section{REFERENCES}

Abraham WC, Gustafsson B, Wigstrom H. 1987. Long-term potentiation involves enhanced synaptic excitation relative to synaptic inhibition in guinea-pig hippocampus. J Physiol 394:367-380. 
Abraham WC, Mason-Parker SE, Logan B. 1996. Low-frequency stimulation does not readily cause long-term depression or depotentiation in the dentate gyrus of awake rats. Brain Res 722:217-221.

Bhat R, Chari G, Rao R. 2006a. Effects of prenatal cocaine, morphine, or both on postnatal opioid $(\mathrm{mu})$ receptor development. Life Sci 78:1478-1482.

Bhat R, Chari G, Rao R, Wirtshafter D. 2006b. Prenatal cocaine and morphine alter brain cyclin-dependent kinase $5(\mathrm{Cdk} 5)$ activity in rat pups. Neurotoxicol Teratol 28:625-628.

Burdette LJ, Gilbert ME. 1995. Stimulus parameters affecting pairedpulse depression of dentate granule cell field potentials. I. Stimulus intensity. Brain Res 680:53-62.

Che Y, Sun H, Tan H, Peng Y, Zeng T, Ma Y. 2005. The effect of prenatal morphine exposure on memory consolidation in the chick. Neurosci Lett 380:300-304.

Chiou LC, Yeh GC, Fan SH, How CH, Chuang KC, Tao PL. 2003. Prenatal morphine exposure decreases analgesia but not $\mathrm{K}+$ channel activation. Neuroreport 14:239-242.

Colicos MA, Dash PK. 1996. Apoptotic morphology of dentate gyrus granule cells following experimental cortical impact injury in rats: Possible role in spatial memory deficits. Brain Res 739:120-131.

Coulter DA, Carlson GC. 2007. Functional regulation of the dentate gyrus by GABA-mediated inhibition. Prog Brain Res 163:235-243.

Dellu F, Fauchey V, Moal ML, Simon H. 1997. Extension of a new two-trial memory task in the rat: Influence of environmental context on recognition processes. Neurobiol Learn Mem 67:112-120.

Dellu F, Contarino A, Simon H, Koob GF, Gold LH. 2000. Genetic differences in response to novelty and spatial memory using a two-trial recognition task in mice. Neurobiol Learn Mem 73:3148.

Errington ML, Bliss TV, Richter-Levin G, Yenk K, Doyere V, Laroche S. 1995. Stimulation at $1-5 \mathrm{~Hz}$ does not produce long-term depression or depotentiation in the hippocampus of the adult rat in vivo. J Neurophysiol 74:1793-1799.

Hol T, Niesink M, van Ree JM, Spruijt BM. 1996. Prenatal exposure to morphine affects juvenile play behavior and adult social behavior in rats. Pharmacol Biochem Behav 55:615-618.

Huang S-M, Mouri A, Kokubo H, Nakajima R, Suemoto T, Higuchi M, Staufenbiel M, Noda Y, Yamaguchi H, Nabeshima T, Saido TC, Iwata N. 2006. Neprilysin-sensitive synapse-associated amyloid-beta peptide oligomers impair neuronal plasticity and cognitive function. J Biol Chem 281:17941-17951.

Kimura A, Pavlides C. 2000. Long-term potentiation/depotentiation are accompanied by complex changes in spontaneous unit activity in the hippocampus. J Neurophysiol 84:1894-1906.

Kirby ML. 1983. Recovery of spinal cord volume in postnatal rats following prenatal exposure to morphine. Brain Res 282:211-217.

Kirby ML, DeRossett SE, Holtzman SG. 1982. Enhanced analgesic response to morphine in adult rats exposed to morphine prenatally. Pharmacol Biochem Behav 17:1161-1164.

Klausnitzer J, Kulla A, Manahan-Vaughan D. 2004. Role of the group III metabotropic glutamate receptor in LTP, depotentiation and LTD in the dentate gyrus of freely moving rats. Neuropharmacology 46:160-170.

Kulla A, Manahan-Vaughan D. 2000. Depotentiation in the dentate gyrus of freely moving rats is modulated by D1/D5 dopamine receptors. Cereb Cortex 10:614-620.

Kulla A, Manahan-Vaughan D. 2002. Modulation by serotonin 5$\mathrm{HT}$ (4) receptors of long-term potentiation and depotentiation in the dentate gyrus of freely moving rats. Cereb Cortex 12:150-162.

Kulla A, Manahan-Vaughan D. 2008. Modulation by group 1 metabotropic glutamate receptors of depotentiation in the dentate gyrus of freely moving rats. Hippocampus 18:48-54.

Kulla A, Reymann KG, Manahan-Vaughan D. 1999. Time-dependent induction of depotentiation in the dentate gyrus of freely moving rats: Involvement of group 2 metabotropic glutamate receptors. Eur J Neurosci 11:3864-3872.
Lomo T. 1971a. Patterns of activation in a monosynaptic cortical pathway: The perforant path input to the dentate area of the hippocampal formation. Exp Brain Res 12:18-45.

Lomo T. 1971b. Potentiation of monosynaptic EPSPs in the perforant path-dentate granule cell synapse. Exp Brain Res 12:46-63.

Lu YM, Mansuy IM, Kandel ER, Roder J. 2000. Calcineurin-mediated ltd of gabaergic inhibition underlies the increased excitability of CA1 neurons associated with LTP. Neuron 26:197-205.

Manahan-Vaughan D, Kulla A. 2003. Regulation of depotentiation and long-term potentiation in the dentate gyrus of freely moving rats by dopamine D2-like receptors. Cereb Cortex 13:123-135.

Marcondes FK, Bianchi FJ, Tanno AP. 2002. Determination of the estrous cycle phases of rats: Some helpful considerations. Braz J Biol 62:6096-6114.

Marder CP, Buonomano DV. 2004. Timing and balance of inhibition enhance the effect of long-term potentiation on cell firing. J Neurosci 24:8873-8884.

Mori K, Togashi H, Ken-ichi U, Matsumoto M, Yoshioka M. 2001. Aminoguanidine prevented the impairment of learning behavior and hippocampal long-term potentiation following transient cerebral ischemia. Behav Brain Res 120:159-168.

Morris RG. 1989. Synaptic plasticity and learning: selective impairment of learning rats and blockade of long-term potentiation in vivo by the $\mathrm{N}$-methyl-D-aspartate receptor antagonist AP5. J Neurosci 9:3040-3057.

Morris RG, Anderson E, Lynch GS, Baudry M. 1986. Selective impairment of learning and blockade of long-term potentiation by an N-methyl-D-aspartate receptor antagonist, AP5. Nature 319:774-776.

Niesink RJ, Vanderschuren LJ, van Ree JM. 1996. Social play in juvenile rats after in utero exposure to morphine. Neurotoxicology 17:905-912.

Niesink RJ, van Buren-van Duinkerken L, van Ree JM. 1999. Social behavior of juvenile rats after in utero exposure to morphine: Dose-time-effect relationship. Neuropharmacology 38:1207-1223.

Nilsson M, Perfilieva E, Johansson U, Orwar O, Eriksson PS. 1999. Enriched environment increases neurogenesis in the adult rat dentate gyrus and improves spatial memory. J Neurobiol 39:569-578.

O'Callaghan JP, Holtzman SG. 1976. Prenatal administration of morphine to the rat: Tolerance to the analgesic effect of morphine in the offspring. J Pharmacol Exp Ther 197:533-544.

Riley MA, Vathy I. 2006. Mid to late gestational morphine exposure does not alter the rewarding properties of morphine in adult male rats. Neuropharmacology 51:295-304.

Robert S, Sloviter GN. 1987. Immunocytochemical localization of GABA-, cholecystokinin-, vasoactive intestinal polypeptide-, and somatostatin-like immunoreactivity in the area dentata and hippocampus of the rat. J Comp Neurol 256:42-60.

Rubin SA, Sylves P, Vogel M, Pletnikov M, Moran TH, Schwartz GJ, Carbone KM. 1999. Borna disease virus-induced hippocampal dentate gyrus damage is associated with spatial learning and memory deficits. Brain Res Bull 48:23-30.

Sarkaki A, Assaei R, Motamedi F, Badavi M, Pajouhi N. 2008. Effect of parental morphine addiction on hippocampal long-term potentiation in rats offspring. Behav Brain Res 186:72-77.

Schindler CJ, Veliskova J, Slamberova R, Vathy I. 2000. Prenatal morphine exposure alters susceptibility to bicuculline seizures in a sexand age-specific manner. Brain Res Dev Brain Res 121:119-122.

Schindler CJ, Slamberova R, Vathy I. 2001. Prenatal morphine exposure decreases susceptibility of adult male rat offspring to bicuculline seizures. Brain Res 922:305-309.

She JQ, Wang M, Zhu DM, Sun LG, Ruan DY. 2005. Effect of ganglioside on synaptic plasticity of hippocampus in lead-exposed rats in vivo. Brain Res 1060:162-169.

Silva AJ, Paylor R, Wehner JM, Tonegawa S. 1992. Impaired spatial learning in alpha-calcium-calmodulin kinase II mutant mice. Science 257:206-211. 
Slamberova R, Schindler CJ, Pometlova M, Urkuti C, Purow-Sokol JA, Vathy I. 2001. Prenatal morphine exposure differentially alters learning and memory in male and female rats. Physiol Behav 73:93-103.

Slamberova R, Rimanoczy A, Bar N, Schindler CJ, Vathy I. 2003. Density of mu-opioid receptors in the hippocampus of adult male and female rats is altered by prenatal morphine exposure and gonadal hormone treatment. Hippocampus 13:461-471.

Slamberova R, Hnatczuk OC, Vathy I. 2004. Expression of proopiomelanocortin and proenkephalin mRNA in sexually dimorphic brain regions are altered in adult male and female rats treated prenatally with morphine. J Pept Res 63:399-408.

Straube T, Frey JU. 2003. Time-dependent depotentiation in the dentate gyrus of freely moving rats by repeated brief $7 \mathrm{~Hz}$ stimulation. Neurosci Lett 339:82-84.

Taube JS, Schwartzkroin PA. 1988. Mechanisms of long-term potentiation: EPSP/spike dissociation, intradendritic recordings, and glutamate sensitivity. J Neurosci 8:1632-1644.

Tomasulo RA, Levy WB, Steward O. 1991. LTP-associated EPSP/ spike dissociation in the dentate gyrus: GABAergic and nonGABAergic components. Brain Res 561:27-34.

UNODC 2006. World Drug Report, 2006. In: United Nations office on Drugs and Crime, editor. United Nations Publications.

van Baar AL, Soepatmi S, Gunning WB, Akkerhuis GW. 1994. Development after prenatal exposure to cocaine, heroin and methadone. Acta Paediatr Suppl 404:40-46.

Vathy I, Katay L. 1992. Effects of prenatal morphine on adult sexual behavior and brain catecholamines in rats. Brain Res Dev Brain Res 68:125-131.

Vathy IU, Etgen AM, Barfield RJ. 1985. Effects of prenatal exposure to morphine on the development of sexual behavior in rats. Pharmacol Biochem Behav 22:227-232.

Vathy I, Rimanoczy A, Eaton RC, Katay L. 1994. Modulation of catecholamine turnover rate in brain regions of rats exposed prenatally to morphine. Brain Res 662:209-215.

Vathy I, Veliskova J, Moshe SL. 1998. Prenatal morphine exposure induces age-related changes in seizure susceptibility in male rats. Pharmacol Biochem Behav 60:635-638.
Vathy I, Slamberova R, Rimanoczy A, Riley MA, Bar N. 2003. Autoradiographic evidence that prenatal morphine exposure sex-dependently alters mu-opioid receptor densities in brain regions that are involved in the control of drug abuse and other motivated behaviors. Prog Neuropsychopharmacol Biol Psychiatry 27:381-393.

Velisek L, Stanton PK, Moshe SL, Vathy I. 2000. Prenatal morphine exposure enhances seizure susceptibility but suppresses long-term potentiation in the limbic system of adult male rats. Brain Res 869:186-193.

Veliskova J, Moshe SL, Vathy I. 1999. Prenatal morphine exposure differentially alters seizure susceptibility in developing female rats. Brain Res Dev Brain Res 116:119-21.

Villarreal DM, Derrick B, Vathy I. 2008. Prenatal morphine exposure attenuates the maintenance of late LTP in lateral perforant path projections to the dentate gyrus and the CA3 region in vivo. J Neurophysiol 99:1235-1242.

Wang M, Chen WH, Zhu DM, She JQ, Ruan DY. 2007. Effects of carbachol on lead-induced impairment of the long-term potentiation/depotentiation in rat dentate gyrus in vivo. Food Chem Toxicol 45:412-418.

Yang SN, Huang LT, Wang CL, Chen WF, Yang CH, Lin SZ, Lai MC, Chen SJ, Tao PL. 2003. Prenatal administration of morphine decreases CREBSerine-133 phosphorylation and synaptic plasticity range mediated by glutamatergic transmission in the hippocampal CA1 area of cognitive-deficient rat offspring. Hippocampus 13: 915-921.

Yang SN, Liu CA, Chung MY, Huang HC, Yeh GC, Wong CS, Lin WW, Yang CH, Tao PL. 2006. Alterations of postsynaptic density proteins in the hippocampus of rat offspring from the morphineaddicted mother: Beneficial effect of dextromethorphan. Hippocampus 16:521-530.

Zhu DM, Wang M, She JQ, Yu K, Ruan DY. 2005. Protection by a taurine supplemented diet from lead-induced deficits of long-term potentiation/depotentiation in dentate gyrus of rats in vivo. Neuroscience 134:215-224.

Zuckerman B, Bresnahan K. 1991. Developmental and behavioral consequences of prenatal drug and alcohol exposure. Pediatr Clin North Am 38:1387-1406. 